\title{
Diagnostic and prognostic relevance of microparticles in peripheral and uterine blood of patients with endometrial cancer
}

\author{
Marek Dziechciowski ${ }^{1}$, Barbara Zapala ${ }^{2}$, Krzysztof Skotniczny ${ }^{3}$, Katarzyna Gawlik ${ }^{2}$, \\ Dorota Pawlica-Gosiewska², Monika Piwowar ${ }^{4}$, Marta Balajewicz-Nowak ${ }^{3}$, \\ Pawel Basta ${ }^{3}$, Bogdan Solnica ${ }^{2}$, Kazimierz Pitynski ${ }^{3}$ \\ ${ }^{1}$ Department of Gynecology and Oncology, University Hospital, Cracow, Poland \\ ${ }^{2}$ Department of Clinical Biochemistry, Jagiellonian University Medical College, Cracow, Poland \\ ${ }^{3}$ Department of Gynecology and Oncology, Jagiellonian University Medical College, Cracow, Poland \\ ${ }^{4}$ Department of Bioinformatics and Telemedicine, Jagiellonian University Medical College, Cracow, Poland
}

\begin{abstract}
Objectives: Exosomes - microvesicles which are secreted by living cells — can be produced from different cell types and detected in various body fluids. They are the carriers of intercellular information which regulate tumor microenvironment and are considered to be involved in tumor progression and metastasis. Cancer cells can secrete more exosomes than healthy cells, and are expected to be potential tools for tumor diagnosis and treatment.

Material and methods: In this report, we present the results of microparticle analysis in peripheral and uterine blood of patients with endometrial cancer. To the best of our knowledge, this study has been the first to report microvesicle status in peripheral and uterine blood samples. The aim of the study was to determine the amount of total (TF+), endothelial (CD144+) and monocytic (CD14+) microparticles. The counting of the selected microparticles in citrate plasma was performed using flow cytometry on the BD Canto II cytometer.

Results: We found that the total amount of microparticles in cancer patients was much higher than in healthy controls. Moreover, microparticle count in uterine blood was higher than in peripheral blood of patients with endometrial cancer. We also demonstrated that the amount of microparticles correlates with the histologic grade and clinical stage of the tumor.

Conclusions: The most interesting finding in this work was the high level of TF, CD144 and CD14 MPs in uterine blood samples. Thus we can consider the monocyte-macrophage-derived MPs as a candidate marker of endometrial cancer and maybe very critical part of the endometrial carcinogenesis.
\end{abstract}

Key words: endometrial cancer; exosomes; microparticles; biomarkers

Ginekologia Polska 2018; 89, 12: 682-687

\section{INTRODUCTION}

Malignant tumors are one of the main factors contributing to high morbidity and mortality rates around the world [1, 2]. Over the next decades, a further increase in tumor-related mortality is expected. The steadily increasing morbidity and mortality rates result, among others, from the extending life expectancy.

Endometrial cancer (EC) is the most common malignant tumor of the female reproductive system in the developed countries. According to the latest data from the National
Register of Tumors, out of 6243 women diagnosed with EC in 2015 in Poland, 1690 died. In the vast majority of cases, EC develops after the menopause and it is diagnosed before the age of 40 only in $5 \%$ of the cases [3].

Old age, diabetes, hypertension and obesity are well-known risk factors for EC. Type I EC develops on the basis of irregular proliferation of the endometrium caused by hormonal stimulation. In contrast, type II EC is hormone-resistant and develops from a thin, atrophic endometrium [4-6]. No screening method allows to detect pre-malignant 
and intraepithelial lesions of the endometrium. Neither Pap smear nor transvaginal ultrasonography constitute sufficient screening criteria. Therefore, the search for a specific biochemical marker, which could be useful in endometrial cancer screening, continues [5-7]. One of the most promising areas of research in modern oncology is the search for biomarkers enabling and facilitating early diagnosis, prognosis and monitoring of cancer treatment.

It has been well-established that cancer progression is largely dependent on abnormal angiogenesis, whereby new vessel formation ensures an adequate supply of nutrients, oxygen, and growth factors to the developing tumor and also facilitates tumor dissemination [8-10]. The results of loss of endothelial cell integrity, dysfunction of the endothelium and prolonged or unregulated activation of the endothelial cells can be observed by the use of specific plasma markers [8-10].

The measurement of the circulating endothelial cells (CEC) level was proposed as a novel marker which could be used to assess vascular function, damaged in cancerous conditions. More recently, endothelial microparticles have been proposed as novel and perhaps more attractive markers which are associated with vascular dysfunction in cancer. These endothelial microparticles (EMP) are vesicles formed by the endothelial cell membrane as a consequence of injury or activation. They are able to harbor cell surface proteins and cytoplasmic elements, thus expressing endothelial specific surface markers which reflect the parent cell status [11, 12].

Microparticles (MPs) are a part of a general category of extracellular vesicles defined as microvesicles (MVs), which includes a population of membrane vesicles. MPs are heterogeneous in shape, ranging in size from 0.1 to $1 \mathrm{um}$. MPs are composed of fragments of the parent cell, which comprise the plasma membrane proteins and cytoplasmic and nucleic constituents of the parent cell $[11,12,13]$. MPs are released into the systemic circulation, where they can effectively deliver their cargo with a long range to recipient cells. Thus, they serve as systemic vehicles in mediating intercellular communication. MPs have been found to carry various bioactive molecules, proteins and nucleic acids, including mRNA and microRNA $[14,15]$. Therefore, they must be involved in multiple aspects of cancer progression, angiogenesis, cellular survival and evasion of the immune surveillance. The presence of circulating endothelial microparticles has recently been recognized as a useful marker of vascular damage and may have a potentially significant role to play in the evaluation of tumor angiogenesis and tumor growth $[8,16]$. Usually absent in the blood of healthy individuals, MPs counts are elevated in diseases hallmarked by the presence of vascular insult, such as neoplastic processes. Circulating endothelial cells are supposed to be the consequence of a particular pathology such as cancer $[13,17]$.
Researches have demonstrated the key role of MPs in the growth, division, and maturation of endothelial precursor cells, important for blood vessel regeneration $[8,9,13,17]$. It has been confirmed that cancer cells secrete more MPs than healthy cells, but the contents of these MPs have been found to be very distinct from each other $[13,17]$. Interestingly, cancer cell-derived microparticles can provide a suitable microenvironment for cancer development and it would be most beneficial to gain a more detailed knowledge about them.

\section{Objectives}

In the present work, we evaluated MPs levels in patients with endometrial cancer and in healthy controls. We hypothesized that MPs count is directly proportional to tumor development. Moreover, we compared MPs counts in peripheral and uterine blood as we expected that there must be an early marker in the uterine cavity which could be detected. We suspected that a higher level of MPs would be found in uterine blood samples. We also investigated the hypothesis that MPs counts could correlate with cancer cells grading (based on histologic grading system, $\mathrm{G}$ ) and clinical stage (based on FIGO staging system).

\section{MATERIAL AND METHODS}

The study was approved by the Jagiellonian University Medical College Ethic Committee. All individuals involved in the study gave their written informed consent. We included $37 \mathrm{pa}-$ tients, aged > 40-75, who were interviewed for their medical history and who had histologically proven endometrial cancer, to the endometrial cancer group. The control group consisted of healthy volunteers ( 23 women aged $>40-65$ years).

Blood was collected on citrate, and centrifuged for $15 \mathrm{~min}$ at $1500 \times \mathrm{g}$ at room temperature. The obtained plasma was briefly centrifuged again for $15 \mathrm{~min}$ at $1500 \mathrm{xg}$ at room temperature and aliquoted into $1.5 \mathrm{~mL}$ tubes and frozen at $-80^{\circ} \mathrm{C}$ until assayed.

\section{Reagents}

The following reagents were used for the determination of the selected MPs: Antibodies against tissue factor with PE (phycoerythrin) (Biolegend, USA) APC (Allophycocyanin) (Biolegend, USA) - marker of all microparticles, Monocyte differentiation antigen also known as CD14 with FITC (fluorescein) (Biolegend, USA) - marker of monocyte microparticles, and vascular endothelial cadherin also known as CD144 with PE (phycoerythrin) (Biolegend, USA) - as a marker for endothelial microparticles. Appropriate isotype control was also used to set the method: mouse antibody lgG1-PE (Biolegend, USA), mouse antibody IgG1- APC (Biolegend, USA), mouse antibody IgM- Pacific Blue (Biolegend, USA). 


\section{The counting of the selected MPs in citrate plasma}

We applied the following procedure: $20 \mu \mathrm{L}$ of plasma sample was added to $80 \mu \mathrm{L}$ of cold PBS. $5 \mu \mathrm{L}$ of Tissue factor - APC and $5 \mu \mathrm{L}$ of the selected antibodies were added to each tube. They were then incubated for $20 \mathrm{~min}$. in the dark at room temperature. After this time, $400 \mu \mathrm{L}$ PBS buffer were added and measured at low flow for $1 \mathrm{~min}$ on a BD Canto Il cytometer.

\section{Statistical analysis}

Based on the manufacturer's flow information $(10 \mu \mathrm{L} / \mathrm{sec})$ and the flow time and dilution of the sample, we know how many microparticles were in patient plasma. Regularity of distribution of the results was assessed using the Kolmogorov-Smirnov test. Non-parametrically distributed results were presented as medians and inter-quartile differences, and medians were compared using the ANOVA Range Kruskal Wallis and U-Mann Whitney test.The $p$-value of $<0.05$ was considered as statistically significant. Spearman correlation coefficient was used to evaluate the correlation.

\section{RESULTS}

The total amount of endogenous MPs was determined and characterized by flow cytometry using tissue factor TF. For identification of endothelial MPs, antibody against hu- man CD144 was used, and for monocytes against CD14. At first, we measured the total amount of circulating MPs in peripheral blood in the control group as compared to EC patients. The total amount of TF+ MPs was much higher in the EC group than controls (3381 [2410-5925]; $\mathrm{P}<0.001$ ) (Tab. 1). The count of cells demonstrating positive CD144+ fluorescence which represented endothelial MPs was seven times higher in peripheral blood of EC patients. The same observation was made with regard to the amount of monocytic MPs. Their count was higher in EC patients than healthy controls (402 [126-3188]; $\mathrm{P}<0.0001)$.

Next, we analyzed the total amount (TF+), endothelial MPs (CD144+) and monocytic (CD14+) in peripheral and uterine blood of patients with EC (Tab. 2). We found that the total amount of circulating MPs was higher in uterine blood (7542 [3687-10745], P < 0.0027), whereas the level of endothelial and monocytic MPs was reduced in uterine blood samples and only for MPs demonstrating positive CD14+ we observed the statistical significance with $\mathrm{P}<0.0251$.

We also demonstrated a correlation between circulating MPs count and histologic grading (G). We distinguished 3 groups of patients with endometrial cancer based on the histologic grading system $\mathrm{G}(\mathrm{G} 1 / \mathrm{G} 2 / \mathrm{G} 3)$. The results are shown in Table 3. MPs counts were different. The highest level of the total MPs was observed in group G3 in uterine blood (7779 [4687-9515]), whereas the values were lower
Table 1. Flow cytometry results for total amount of circulating and endothelial MPs and monocytic MPs in peripheral blood of patients with endometrial cancer as compared to the control group

\begin{tabular}{|l|l|l|l|}
\hline & $\begin{array}{l}\text { CONTROL } \\
\text { GROUP, N }=23\end{array}$ & $\begin{array}{l}\text { ENDOMETRIAL } \\
\text { CANCER, N = 37 } \\
\text { (peripheral blood) }\end{array}$ & P \\
\hline TF+ & $435[345-592]$ & $3381[2410-5925]$ & $<0.0001$ \\
\hline TF+CD144+ & $116[80-142]$ & $749[476-1275]$ & $<0.0001$ \\
\hline TF+CD14+ & $82[57-95]$ & $402[126-3188]$ & $<0.0001$ \\
\hline
\end{tabular}

Total amount of MPs is defined as TF+, whereas endothelial MPs are characterized by $\mathrm{CD} 144+$ and monocytic MPs by CD14+

\begin{tabular}{l} 
Table 2. Flow cytometry results for total amount of circulating and \\
endothelial MPs and monocytic in patients with endometrial cancer \\
\hline \\
\begin{tabular}{|l|l|l|l|}
\hline & $\begin{array}{l}\text { ENDOMETRIAL } \\
\text { CANCER, N=37 } \\
\text { (peripheral blood) }\end{array}$ & $\begin{array}{l}\text { ENDOMETRIAL } \\
\text { CANCER, N = 37 } \\
\text { (uterine blood) }\end{array}$ & P \\
\hline TF+ & $3381[2410-5925]$ & $7542[3687-10745]$ & 0.0027 \\
\hline TF+CD144+ & $749[476-1275]$ & $484[268-1154]$ & 0.1652 \\
\hline TF+CD14+ & $402[126-3188]$ & $252[139-453]$ & 0.0251 \\
\hline
\end{tabular}
\end{tabular}

Total amount of MPs is defined as TF+, whereas endothelial MPs are characterized by CD144+ and monocytic by CD14+. Comparison of MPs level in peripheral blood and uterine blood samples from the same patients with endometrial cancer

Table 3. Flow cytometry results for total amount of circulating and endothelial MPs and monocytic in patients with endometrial cancer

\begin{tabular}{|l|l|l|l|l|}
\hline & $\mathbf{G 1}, \mathbf{N}=\mathbf{1 5}$ & $\mathbf{G 2}, \mathbf{N}=\mathbf{1 3}$ & $\mathbf{G 3 , \mathbf { N } = \mathbf { 9 }}$ \\
\hline TF+ & $2714[2343-6316]$ & $3672[2683-5080]$ & $3533[1681-15259]$ & $\mathbf{P}$ \\
\hline TF+CD144+ & $731[464-842]$ & $1123[636-1864]$ & $582[476-1290]$ & 0.8830 \\
\hline TF+CD14+ & $319[224-543]$ & $451[252-556]$ & $348[294-628]$ & 0.4927 \\
\hline UTF+ & $7555[2253-13496]$ & $6484[3687-10745]$ & $7779[4687-9515]$ & 0.8627 \\
\hline UTF+CD144+ & $613[265-1494]$ & $471[273-1154]$ & $484[268-600]$ & 0.9163 \\
\hline UTF+CD14+ & $363[129-577]$ & $252[155-412]$ & $201[134-283]$ & 0.6661 \\
\hline
\end{tabular}

Total amount of MPs is defined as TF+, whereas endothelial MPs are characterized by CD144+ and monocytic by CD14+. Comparison of MPs level based on histologic grading (G). (ANOVA Range Kruskal Wallis). $U$ is defined for results in uterine blood samples 
Table 4. Flow cytometry results for total amount of circulating and endothelial MPs and monocytic in patients with endometrial cancer

\begin{tabular}{|l|l|l|l|}
\hline & FIGO I, N = 27 & FIGO $>$ III, N = 9 & P \\
\hline TF+ & $3180[2343-5925]$ & $3533[2683-3968]$ & 0.970857 \\
\hline TF+CD144+ & $731[464-986]$ & $1190[520-1290]$ & 0.323941 \\
\hline TF+CD14+ & $348[252-543]$ & $531[219-628]$ & 0.583695 \\
\hline UTF+ & $755[[4498-12208]$ & $6484[3206-10745]$ & 0.714865 \\
\hline UTF+CD144+ & $525[296-1236]$ & $363[173-994]$ & 0.235100 \\
\hline UTF+CD14+ & $258[147-543]$ & $167[129-453]$ & 0.411080 \\
\hline
\end{tabular}

Total amount of MPs is defined as TF+, whereas endothelial MPs are characterized by CD144+ and monocytic by CD14+. Comparison of MPs level based on clinical status of endometrial cancer (based on FIGO-grade) (U Mann Whitney). $U$ is defined for results in uterine blood samples

\begin{tabular}{|c|c|c|c|c|c|c|}
\hline Variables & TF+ & TF+CD144+ & $\mathrm{TF}+\mathrm{CD} 14+$ & UTF+ & UT F+CD144+ & U TF+CD14+ \\
\hline TF+ & 1.000000 & 0.833194 & 0.820309 & 0.397108 & 0.363108 & 0.363108 \\
\hline TF+CD144+ & 0.833194 & 1.000000 & 0.947502 & -0.163348 & 0.124948 & 0.124948 \\
\hline TF+CD14+ & 0.820309 & 0.947502 & 1.000000 & 0.022645 & 0.230792 & 0.230792 \\
\hline U TF+ & 0.397108 & -0.163348 & 0.022645 & 1.000000 & 0.650821 & 0.650821 \\
\hline UTF+CD144+ & 0.363108 & 0.124948 & 0.230792 & 0.650821 & 1.000000 & 1.000000 \\
\hline U TF+CD14+ & 0.342640 & 0.105045 & 0.191628 & 0.674017 & 0.917062 & 0.917062 \\
\hline
\end{tabular}

Total amount of MPs is defined as TF+, endothelial MPs are characterized by CD144+ and monocytic by CD14+. Correlations between MPs were evaluated using Spearman correlation coefficients $(r=0.78,0.87$, and 0.90 , respectively; all $P<0.05000)$. $U$ is defined for results in uterine blood samples

for CD144+ and CD14+ MPs in uterine blood, especially in group $\mathrm{G} 3$. However, due to a very low number of samples ( $N=15$ for $\mathrm{G} 1, \mathrm{~N}=13$ for $\mathrm{G} 2$ and only $\mathrm{N}=9$ for $\mathrm{G} 3$ ), no statistically significant trends were observed for this step of the performed analysis.

In Table 4, we presented two-fold higher numbers of the total MPs in uterine blood for FIGO I stage of endometrial cancer in comparison to the MPs count in peripheral blood. The lower CD144+ and CD14+ MPs count was observed in uterine blood samples both, for FIGO I and FIGO > III stage of endometrial cancer. We were not able to confirm these results statistically due to the low and not equal number of samples from FIGO I and FIGO > III groups.

In Table 5, Spearman correlation coefficients between MPs count (total, endothelial and monocytic) are shown. We found significant correlations for the total MPs count $(\mathrm{TF}+)$ and the endothelial (TF+CD144+) and monocytic (TF+CD14+) MPs. Moreover, we found correlations between endothelial and monocytic MPs both, in peripheral and uterine blood of patients with endometrial cancer.

\section{DISCUSSION}

Endothelial homeostasis is crucial in many pathological conditions, including vascular damage, regeneration and adult neovasculogenesis. In many recent studies, it has been demonstrated that MPs may reflect the endothelium status in both, health and disease $[10,13,17]$. In human blood, circulating endothelial MPs have been widely associated with endothelial dysfunction, suggesting a potential preservative role in response to vascular regeneration, restoration, and protection. It is important to identify differences in the level of MPs in healthy and pathological conditions. Clinical applications of membrane vesicles are still in the developmental stage and their full potential is yet to be explored. Despite numerous studies on the role of MPs in cancer pathogenesis, the number of reports on their role in endometrial cancer remains limited. High levels of MPs in cancer have been reported but in many cases those levels fail to be associated with progression and staging $[13,17]$. Newly demonstrated ways of assessing vascular function in the treatment of cancer include the measurement of levels of CEC and circulating endothelial microparticles. An increased MPs count in peripheral blood reflects significant vascular damage and dysfunction. Recently, an interest in the evaluation of the endothelial-specific adhesion molecule VE-cadherin (cadherin-5 or CD144), specifically in the context of tumor angiogenesis, has increased $[8,9]$. It has been proven that monocytic molecules activate endothelial cells. In addition, it has been demonstrated that there are very close correlations between endothelium-derived and monocytic-derived microparticles. In our study, we found higher levels of the total and endothelial MPs as well as monocytic in the peripheral blood samples from the patients 
with endometrial cancer. Our findings are in accordance with other studies performed to date. The levels of TF+, CD144+ and CD14+ MPs in uterine blood samples are a particularly interesting and innovative finding of our study. To the best of our knowledge, this has been the first report concerning MPs in the uterine blood of patients with endometrial cancer. We demonstrated that the total TF+ MPs count was higher in uterine blood in comparison to peripheral blood. The quantity of endothelial and monocytic MPs was lower but two-fold higher numbers for CD144+ positive microparticles in comparison with CD14+ were found.

We were slightly surprised to find that the levels of MPs were neither related to the clinical stage (FIGO) nor to the histologic grading (based on $\mathrm{G}$ ) of the tumor. We suppose that the reason for that could be lack of statistical power due to a relatively small sample size in our study, although it may also reflect pathophysiological processes. The highest level of total MPs in uterine blood as compared to the peripheral blood samples as well as to the healthy group suggested that there must be some unspecific cells which we were not able to identify using CD144 and CD14 markers, or that there are some unknown pathophysiological changes underlying this type of cancer. In human blood, endothelial MPs constitute a smaller population of MPs family. However, they are associated with the pathogenesis of various cardiovascular diseases, mainly initiated by endothelial dysfunction [8]. In our study, we found that monocytic MPs (CD14+) may increase endothelial MPs (CD144+) count, and what is very interesting, that there were many correlations between monocytic and endothelial MPs, which may indicate their similar functions in pathological conditions. What is more, the analysis of endothelial and monocytic MPs in correlation with the histologic grading and clinical stage of endometrial cancer yielded higher levels of CD144+ and CD14+ particles in G1 and FIGO I in comparison to G3 and FIGO > III. These results may indicate that in a histologically advanced and late clinical stage of cancer there is a higher accumulation of dead cells. However, it can also mean that MPs could serve as a marker for early detection of endometrial cancer, but further studies are needed.

In the future studies, we should focus more on the samples from the uterine cavity as an organ where markers may appear earlier than in the peripheral blood. We suggest that microparticles in the uterine blood should be characterized in detail. Particularly, we should be more focused on their involvement in molecular processes in which cancer-related MPs could be involved. We are aware that there are several limitations of this study. Despite our best efforts to minimize the confounding factors by careful screening of the patients and controls, it is possible that some factors have not been adequately verified. However, our data suggest that MPs may be considered as a candidate biomarker for early detection of endometrial cancer. It seems prudent to perform additional studies, concentrating on the uterine cavity where the endometrial cancer proliferation starts. Endothelial MPs release is triggered by various stimuli followed by the activation of various pathways which can be involved in carcinogenesis. As we know, many factors such as oxidative stress, endogenous molecules and apoptosis inducer are responsible for endothelial MPs release to undergo a functional change in the blood vessel. The monocyte-macrophage-derived MPs may also play a role in the progression of endometrial cancer. It is worth noting that medicines targeting the downregulation of endothelial, monocytic or other MPs release can be a possible therapeutic option for treating endometrial or other gynecological tumors.

\section{CONCLUSIONS}

Endothelial microparticles release is triggered by various stimuli, followed by the activation of various pathways which can collectively make progress in the endometrial cancer advancement. Moreover, monocyte-macrophage-derived MPs may also be a very critical part of the endometrial carcinogenesis. According to our data, the total amount of microparticles in cancer patients is much higher than in healthy subjects. In uterine blood, the count of microparticles is higher than in peripheral blood of patients with endometrial cancer. MPs count in uterine blood can be considered as a candidate marker of endometrial cancer and detailed evaluation of MPs continues to present a big challenge, especially to discover their clinical utility.

\section{Acknowledgements}

This work was funded by Jagiellonian University Medical College and by the Ministry of Science and Higher Education; grant no. K/ZDS/007951.

\section{REFERENCES}

1. Lemech CR, Ensell L, Paterson JC, et al. Enumeration and Molecular Characterisation of Circulating Tumour Cells in Endometrial Cancer. Oncology. 2016; 91(1): 48-54, doi: 10.1159/000445999, indexed in Pubmed: 27256106.

2. Li XY, Wang X. The role of human cervical cancer oncogene in cancer progression. Int J Clin Exp Med. 2015; 8(6): 8363-8368, indexed in Pubmed: 26309489.

3. Colombo N, Creutzberg C, Amant F, et al. ESMO-ESGO-ESTRO Endometrial Consensus Conference Working Group. ESMO-ESGO-ESTRO consensus conference on endometrial cancer: Diagnosis, treatment and follow-up. Radiother Oncol. 2015; 117(3): 559-581, doi: 10.1016/j. radonc.2015.11.013, indexed in Pubmed: 26683800.

4. Kandoth C, Schultz N, Cherniack AD, et al. Cancer Genome Atlas Research Network; Integrated Genomic Characterization of Endometrial Carcinoma. Nature. 2013; 497: 67-73.

5. Piulats JM, Guerra E, Gil-Martín M, et al. Molecular approaches for classifying endometrial carcinoma. Gynecol Oncol. 2017; 145(1): 200-207, doi: 10.1016/j.ygyno.2016.12.015, indexed in Pubmed: 28040204.

6. Talhouk A, Hoang LN, McConechy MK, et al. Molecular classification of endometrial carcinoma on diagnostic specimens is highly concordant with final hysterectomy: Earlier prognostic information to guide treatment. Gynecol Oncol. 2016; 143(1): 46-53, doi: 10.1016/j.ygyno.2016.07.090, indexed in Pubmed: 27421752

7. van Niel G, D'Angelo G, Raposo G. Shedding light on the cell biology of extracellular vesicles. Nat Rev Mol Cell Biol. 2018; 19(4): 213-228, doi: 10.1038/nrm.2017.125, indexed in Pubmed: 29339798. 
8. Goon PKY, Lip GYH, Stonelake PS, et al. Circulating endothelial cells: markers of vascular dysfunction. Clin Lab. 2005; 51(9-10): 531-538, indexed in Pubmed: 16285476.

9. Kim CW, Lee HM, Lee TH, et al. Extracellular membrane vesicles from tumor cells promote angiogenesis via sphingomyelin. Cancer Res. 2002; 62(21): 6312-6317, indexed in Pubmed: 12414662.

10. Mancuso $P$, Calleri A, Cassi C, et al. Circulating endothelial cells as a novel marker of angiogenesis. Adv Exp Med Biol. 2003; 522: 83-97, indexed in Pubmed: 12674213.

11. Lin $Y$, Weisdorf DJ, Solovey $A$, et al. Origins of circulating endothelial cells and endothelial outgrowth from blood. J Clin Invest. 2000; 105(1): 71-77, doi: 10.1172/JCl8071, indexed in Pubmed: 10619863.

12. Ura B, Monasta L, Arrigoni G, et al. A proteomic approach for the identification of biomarkers in endometrial cancer uterine aspirate. Oncotarget. 2017; 8(65): 109536-109545, doi: 10.18632/oncotarget.22725, indexed in Pubmed: 29312627.
13. Beerepoot LV, Mehra N, Vermaat JSP, et al. Increased levels of viable circulating endothelial cells are an indicator of progressive disease in cancer patients. Ann Oncol. 2004; 15(1): 139-145, indexed in Pubmed: 14679134.

14. Gong J, LukF, Jaiswal R, et al. Microparticles Mediate the Intercellular Regulation of microRNA-503 and Proline-Rich Tyrosine Kinase 2 to Alter the Migration and Invasion Capacity of Breast Cancer Cells. Front Oncol. 2014; 4: 220, doi: 10.3389/fonc.2014.00220, indexed in Pubmed: 25177548.

15. Sun $D, Z$ Zhuang $X$, Zhang $S$, etal. Exosomes are endogenous nanoparticles that can deliver biological information between cells. Adv Drug Deliv Rev. 2013; 65(3):342-347, doi: 10.1016/j.addr.2012.07.002, indexed in Pubmed: 22776312.

16. Jaiswal R, Gong J, Sambasivam S, et al. Microparticle-associated nucleic acids mediate trait dominance in cancer. FASEB J. 2012; 26(1): 420-429, doi: 10.1096/f.11-186817, indexed in Pubmed: 21965597.

17. Mancuso P, Burlini A, Pruneri G, et al. Resting and activated endothelial cells are increased in the peripheral blood of cancer patients. Blood. 2001; 97(11): 3658-3661, indexed in Pubmed: 11369666. 